\title{
SEQUENCING BAT GENOMES TO SHED LIGHT ON LANGUAGE EVOLUTION
}

\author{
KSENIA LAVRICHENKO ${ }^{* 1}$, SONJA C. VERNES ${ }^{1,2}$ \\ *Corresponding Author: ksenia.lavrichenko@mpi.nl \\ ${ }^{1}$ Neurogenetics of Vocal Communication Group, Max Planck Institute for \\ Psycholinguistics, Nijmegen, The Netherlands \\ ${ }^{2}$ Donders Centre for Cognitive Neuroimaging, Nijmegen, The Netherlands
}

\section{Summary}

Language is characterized by a number of biological traits, many of which are unique to humans, however some are shared with a handful of other species. These shared traits present an opportunity for language researchers to use cutting-edge methods on relevant model species to study the biological underpinnings of language and its evolution. One such shared, language-relevant trait is vocal learning; the ability to change or create completely new sounds based on auditory feedback (Janik \& Slater, 2000; Petkov \& Jarvis, 2012).

Vocal learning birds are the most well studied species, and recent efforts to perform large scale sequencing of bird genomes has led to advances in our understanding of how this trait is encoded in a genome (Pfenning et al., 2014). Similarly it has opened the door to molecular and neurogenetic interrogations in both learning and non-vocal learning species (Haesler et al., 2007; Whitney et al, 2014) that will help demonstrate what is necessary and/or sufficient for the evolution of a vocal learning brain/animal.

To gain a broader view of how genomes evolved to facilitate vocal learning, it would be of great value to address such questions in other, more distantly related vocal learning species. Bats have recently caught the attention of the field due to their potential for vocal learning, sophisticated social structure and diversity (Knörnschild, 2014). Two species in particular have recently shown promise for vocal learning; Phyllostomus discolor (Esser, 1994) and Rousettus 
aegypticaus (Prat et al., 2015). Studying the genomes of these species provides a great opportunity to pinpoint genetic loci and major evolutionary events that may be relevant for the emergence of vocal learning in mammals. Furthermore, this will facilitate in depth molecular and neurogenetic studies to understand the biological encoding of this trait in a mammalian brain.

We will report our work using existing and newly generated sequence data to assemble complete genomes and transcriptomes for both these species and the potential it has to answer wider queries about the encoding and evolution of vocal learning. With such data at hand, a number of questions can be addressed, such as:

1. What are the shared features of a vocal learning genome, i.e. what are the sequence evolution patterns within the genetic loci relevant to vocal learning both within bat genomes and between bats and other groups, such as birds?

2. Is there evidence of functional evolution in these loci, taken into account the higher than expected sequence variability in bats compared to other species? (Li et al., 2007)

3. What are the patterns of gene expression in vocal learning-relevant regions of the brain?

4. What are the genes and gene networks that play role in vocal learning in the brains of these bats? (Rodenas-Cuadrado et al., 2015).

Studies addressing these and follow up questions will build a sound foundation for elucidating molecular and genetic bases of vocal learning in bats and consequently in humans, as well as providing hints on how this trait emerges in evolution.

\section{Ethical statement}

All tissue was obtained from captive colony animals that were kept in compliance with the principles of laboratory animal care and the regulations of the relevant national laws for animal Protection.

\section{Acknowledgements}

This work was funded by a Marie Curie Career Integration Grant (PCIG12-GA2012-333978), and a Max Planck Research Group Grant both awarded to S.C.V. This work was also funded by a Human Frontiers Science Program (HFSP) Research grant (RGP0058/2016) and is part of a collaborative project involving Prof. Lutz Wiegrebe (LMU, Germany), Prof Michael Yartsev (UC Berkeley, USA) and Prof Uwe Firzlaff (TUM, Germany). With thanks to Prof Erich D. 
Jarvis (Rockefeller University, USA), the G10k Vertebrate Genome Laboratory and Olivier Fedrigo (Rockefeller University, USA) for sequencing and assembling the genomes; and to the directors of Bat1k consortium for their contributions to this project.

\section{References}

Janik, V. and Slater, P. (2000). The different roles of social learning in vocal communication. Animal Behaviour, 60(1), pp.1-11.

Petkov, C. and Jarvis, E. (2012). Birds, primates, and spoken language origins: behavioral phenotypes and neurobiological substrates. Frontiers in Evolutionary Neuroscience, 4.

Pfenning, A., Hara, E., Whitney, O., Rivas, M., Wang, R., Roulhac, P., Howard, J., Wirthlin, M., Lovell, P., Ganapathy, G., Mountcastle, J., Moseley, M., Thompson, J., Soderblom, E., Iriki, A., Kato, M., Gilbert, M., Zhang, G., Bakken, T., Bongaarts, A., Bernard, A., Lein, E., Mello, C., Hartemink, A. and Jarvis, E. (2014). Convergent transcriptional specializations in the brains of humans and song-learning birds. Science, 346(6215), pp.12568461256846.

Haesler, S., Rochefort, C., Georgi, B., Licznerski, P., Osten, P. and Scharff, C. (2007). Incomplete and Inaccurate Vocal Imitation after Knockdown of FoxP2 in Songbird Basal Ganglia Nucleus Area X. PLoS Biology, 5(12), p.e321.

Whitney, O., Pfenning, A., Howard, J., Blatti, C., Liu, F., Ward, J., Wang, R., Audet, J., Kellis, M., Mukherjee, S., Sinha, S., Hartemink, A., West, A. and Jarvis, E. (2014). Core and region-enriched networks of behaviorally regulated genes and the singing genome. Science, 346(6215), pp.12567801256780 .

Knörnschild, M. (2014). Vocal production learning in bats. Current Opinion in Neurobiology, 28, pp.80-85.

Esser, K. (1994). Audio-vocal learning in a non-human mammal. NeuroReport, 5(14), pp.1718-1720.

Prat, Y., Taub, M. and Yovel, Y. (2015). Vocal learning in a social mammal: Demonstrated by isolation and playback experiments in bats. Science Advances, 1(2), pp.e1500019-e1500019.

Li, G., Wang, J., Rossiter, S., Jones, G. and Zhang, S. (2007). Accelerated FoxP2 Evolution in Echolocating Bats. PLoS ONE, 2(9), p.e900.

Rodenas-Cuadrado, P., Chen, X., Wiegrebe, L., Firzlaff, U. and Vernes, S. (2015). A novel approach identifies the first transcriptome networks in bats: a new genetic model for vocal communication. BMC Genomics, 16(1). 